Rodríguez Rodríguez, D. y Guzmán Rosquete, R. (2019). Rendimiento académico de adolescentes declarados en situación de riesgo. Revista de Investigación Educativa, 37(1), 147-162.

DOI: http://dx.doi.org/10.6018/rie.37.1.303391

\title{
Rendimiento académico de adolescentes declarados en situación de riesgo
}

\section{Academic achievement of adolescents declared at risk}

\author{
Daniel Rodríguez Rodríguez y Remedios Guzmán Rosquete \\ Departamento de Didáctica e Investigación Educativa, Universidad de La Laguna, San Cristóbal de La Laguna, España
}

\section{Resumen}

La reciente regulación legal y social de la infancia y la adolescencia en situación de riesgo en España hace que sea necesario la generación de un cuerpo de investigación que desde el ámbito de la educación fomente factores protectores y favorezca el bienestar de los menores, ya que a pesar de la heterogeneidad que caracteriza a esta población, el fomento de su éxito educativo será sin duda la mejor medida preventiva de la situación de riesgo. La muestra fue de 232 estudiantes declarados en situación de riesgo, con bajo (115 estudiantes) y buen rendimiento académico (117 estudiantes) escolarizados en Educación Secundaria, de los que se recogió información sobre variables personales y sociofamiliares que guardan relación con el rendimiento. En relación a las variables personales, los resultados mostraron que los alumnos que tienen un buen rendimiento académico se caracterizan por tener mayores puntuaciones en sus metas académicas, atribuyen su éxito académico a su propia capacidad y esfuerzo y perciben un mayor apoyo familiar afectivo de su familia. Respecto a las variables familiares, se encontró que los alumnos que tienen un buen rendimiento se caracterizan por tener un mayor porcentaje de padres con titulación universitaria, padres y madres que trabajan como funcionarios y madres que esperan que su hijo o hija alcance una titulación universitaria. Como conclusión se confirma la importancia del trabajo conjunto desde los ámbitos educativo y social para prevenir el fracaso escolar.

Palabras clave: situación de riesgo; Educación Secundaria Obligatoria; rendimiento académico; factores personales; contexto sociofamiliar.

Correspondencia: Daniel Rodríguez Rodríguez, drodriguez@gruposhine.com, Departamento de Didáctica e Investigación Educativa, Universidad de La Laguna, San Cristóbal de La Laguna, España. 


\begin{abstract}
The recent legal and social regulation of childhood and adolescence at risk in Spain makes it necessary to create a body of research that encourages, from the field of education, protective factors and promotes the wellbeing of children, because despite of the heterogeneity that characterizes this population, the promotion of educational success will undoubtedly be the best preventive measure of the risk situation. The sample was of 232 students declared at risk, with students of low (115 ) and good academic achievement (117) enrolled in Secondary Education, of which we collected information about personal and family variables that are related to achievement. Regarding the personal variables, results showed that students that have a good academic achievement are characterized by higher scores in their academic goals, they attribute their academic success to their own capacity and effort and they perceive a greater affective family support from their family. Regarding family variables, we found that students with good academic achievement have a higher percentage of parents with a university degree, parents who work in the public sector and mothers who expect that their son or daughterwill achieve a university degree. In conclusion, the importance of working together from the educational and social levels to prevent school failure is confirmed.

Keywords: risk situation; compulsory secondary education; academic achievement; personal factors; socio-familial context.
\end{abstract}

\title{
Introducción
}

La relación rendimiento académico y las variables, personales, escolares y familiares que pueden influir en el mismo ha sido un tópico ampliamente investigado. Sin embargo, la mayor parte de estos estudios se han centrado en poblaciones escolares que, aun siendo diversa, no se encuentran en una situación de riesgo declarado, lo cual los convierte en más vulnerable a la hora de obtener un buen rendimiento académico o terminar la Educación Secundaria Obligatoria (E.S.O.). Quizás la ausencia de estudios sobre los menores declarados en situación de riesgo, sea debido a no fue hasta 2015 cuando se produce en España un hito histórico con la aprobación de la Ley Orgánica 8/2015, de 22 de julio, y la Ley 26/2015, de 28 de julio, de Modificación del Sistema de Protección a la Infancia y a la Adolescencia. Estas normativas han permitido a nivel de todo el territorio español la unificación, principalmente jurídica y social, de la situación de riesgo con la que algunos menores pueden ser declarados. Situación jurídica-administrativa que, sin alcanzar la intensidad que fundamentaría una situación de desamparo, algunos menores pueden encontrar obstáculos para su desarrollo integral, pudiéndose ver perjudicado su desarrollo personal, familiar, social o educativo.

Si bien, el disponer de un marco de referencia común ha permito garantizar una interpretación más homogénea sobre la situación de riesgo, el término en sí mismo alude a un colectivo muy heterogéneo, ya que normalmente las causas familiares o sociales que generan esta situación son muy dispares (pobreza, deprivación, negligencia familiar, problemas de conducta, maltrato, movimientos migratorios, comisión de delitos, etc.). Todas estas causas han sido asociadas, desde la investigación, a factores que afectan negativamente al rendimiento académico (Martínez \& Álvarez, 2005; Rogero, 2012), por lo que resulta de máximo interés realizar un análisis exhaustivo sobre la incidencia que pueden tener en estos estudiantes durante su etapa educativa. Estos factores de riesgo 
normalmente no se dan de manera aislada, sino que suelen presentarse asociados y de forma acumulativa, siendo en estos últimos casos mayor su impacto en el rendimiento académico (Buehler \& Gerard, 2013; Jackson, Ajayi y Quigley, 2003; Roy y Raver, 2014). Así, por ejemplo, la pobreza y los bajos ingresos económicos en la familia han sido relacionados con el bajo rendimiento académico y el abandono escolar temprano (McLoyd et al., 2009; Schoon et al., 2002; Serna, Yubero \& Larrañaga, 2008; Whipple, Evans, Barry \& Maxwell, 2010). En esta dirección, el estudio de Stein y Munro (2008), realizado con información recogida de dieciséis países distintos y tan diversos como Canadá, Rumanía, Jordania o Noruega, mostró que los jóvenes declarados en situación de riesgo por deprivación y negligencia familiar eran los que presentaban mayores tasas de abandono del sistema educativo. Además, en estos estudios se ha encontrado que cuando no se interviene lo más pronto posible sobre el conjunto de estos factores de riesgo los efectos perjudiciales o negativos sobre el rendimiento se mantienen e incrementan en el tiempo.

En relación con el rendimiento académico, aunque son numerosos los factores que pueden influir directa o indirectamente en los resultados académicos, en este estudio se han considerado algunos de los factores personales y sociofamiliares que han mostrado diferenciar entre estudiantes con bajo y normal o buen rendimiento académico. Al respecto, los modelos explicativos sobre el rendimiento han evidenciado que determinadas variables personales, como la motivación y las atribuciones causales, ejercen una influencia decisiva en los resultados académicos. Así en diversos trabajos se ha resaltado la relación directa que tiene la motivación en el rendimiento (Deci \& Ryan, 1985; Dweck y Elliot, 1983; Entwistle \& Kozéki, 1985; McKenzie, Gow \& Schweitzer, 2004; Pintrich \& De Groot, 1990; Rao, Moely \& Sachs, 2000). Resultan de interés los estudios centrados en las metas académicas como factor protector relevante en la prevención del fracaso escolar o abandono prematuro de los estudios obligatorios (Alderman, 2008; Meece, Anderman \& Anderman, 2006). Respecto a las atribuciones causales se ha demostrado que aquellas atribuciones más asociadas a un locus de control interno suponen un factor protector en situaciones de riesgo para jóvenes y adolescentes (Benzies \& Mychasiuk, 2009; Ross \& Mirowsky, 2013), así como determinantes de la motivación, ya que la tendencia a lograr el éxito y evitar el fracaso en una situación escolar concreta depende de las causas a las que se atribuyen dichos éxitos y fracasos (Naranjo, 2009). Existe, por tanto, una relación de incidencia de las atribuciones causales sobre las metas académicas (Cabanach et al., 2009; Closas, Sanz \& Ugarte, 2011).

Si importancia tiene considerar las variables personales no menos recobra, sobre todo por la situación de riesgo de los estudiantes de este estudio, identificar algunos de los factores sociofamiliares que pueden estar en la base de las diferencias del rendimiento de este alumnado. Ello resulta de relevancia si se quiere que la familia se convierta en un activo dentro de la escolaridad y contribuya a ser, igualmente, un factor protector para el óptimo desarrollo de estos menores. De hecho, en el estudio realizado por Oliva, Jiménez, Parra y Sánchez-Queija (2008) se ha podido comprobar que adolescentes resilientes gozaban de relaciones familiares de mayor calidad que los adolescentes mal adaptados. Además, como concluyen estos autores, de entre las variables familiares que inciden en el rendimiento académico, las expectativas de los padres respecto al logro académico de sus hijos han recibido el soporte de la investigación social y educativa para ser considerada una variable influyente. Principalmente, por la incidencia que pueden ejercer las expectativas de los padres en la motivación y estimulación del rendimiento de sus hijos (Ames \& Archer, 
1987; Benner \& Mistry, 2007), de manera especial durante su escolarización en la etapa de Educación Secundaria. Se ha encontrado que estas expectativas no solo son relevantes en la adolescencia, sino que su efecto perdura en el tiempo, incluso hasta edades posteriores (Gordon \& Cui, 2012). Los estudios han demostrado que los menores que son animados por sus padres para esforzarse académicamente y alcanzar mayores cotas educativas tendían a obtener mejores resultados escolares (Chen \& Gregory, 2009), además de influir en la mejora del logro académico a lo largo del tiempo (Mistry, White, Benner \& Huynh, 2009).

Cabe destacar que las expectativas de los padres no solo son importantes en los resultados académicos y en la motivación por aprender, sino que igualmente las percepciones que los estudiantes tienen de su clima y apoyo familiar ejercen una influencia significativa en el rendimiento escolar (Córdoba-Caro, García, Luengo, Vizuete \& Feu, 2011; DavisKean, 2005; Yamamoto \& Holloway, 2010). El nivel educativo de los progenitores ha sido identificado como otro de los factores familiares que incide en el logro académico de los hijos (Brown \& Iyengar, 2008; De la Orden \& González, 2005; Spera, Wentzel \& Matto, 2009). Estudios como el de Gordon y Cui (2012) han revelado que los hijos de padres y madres con estudios universitarios tendían a tener un mejor rendimiento académico que los hijos de los padres y madres con estudios académicos inferiores.

Hasta el momento, desde las nuevas leyes que desde el año 2015 regulan el sistema de protección a la infancia y a la adolescencia en España, pocas investigaciones se han realizado sobre los menores en situación de riesgo. De ahí la importancia de realizar estudios como el presente que permitan identificar las variables que pueden actuar como protectoras de la situación de riesgo en la que se encuentran algunos estudiantes y, en consecuencia, contribuyan a llevar a cabo intervenciones que incrementen las posibilidades de éxito escolar de estos estudiantes.

\section{Objetivos}

Este estudio se planteó con el objetivo de analizar las relaciones entre variables individuales y sociofamiliares en estudiantes de la E.S.O., declarados en situación de riesgo, con bajo y normal rendimiento, e identificar las diferencias que se producen en las variables analizadas en función del rendimiento académico.

\section{Método}

\section{Población y Muestra}

Dada las características de la población objeto de estudio, se llevó a cabo un muestreo no probabilístico de tipo incidental.

La muestra estuvo formada por 232 estudiantes (124 chicos y 108 chicas) declarados en situación de riesgo, según los datos de los Servicios Sociales de 12 municipios de Tenerife. Los menores tenían un rango de edad entre los 12 y los 17 años $(\mathrm{M}=14.26$; DT=1.15) y estaban escolarizados en la E.S.O. El 27.6\% de estos estudiantes cursaban primer curso, el $31.5 \%$ segundo, el $24.1 \%$ tercero y el $16.8 \%$ cuarto de la E.S.O.

La muestra total se dividió, en función del rendimiento académico, en dos grupos: uno formado por estudiantes declarados en riesgo con bajo rendimiento académico 
(BR) y el otro, igualmente formado por estudiantes en situación de riesgo, pero con un rendimiento normal o bueno (NR). El criterio utilizado para determinar el grupo de BR fue tener suspendidas tres o más materias, o las materias básicas de Lengua Castellana y Matemáticas de forma simultánea, ya que es el criterio recogido en la LOMCE (2013) para la repetición de curso en esta etapa educativa. En el grupo de NR se incluyeron todos aquellos estudiantes de la muestra que no cumplían las condiciones para repetir y, por tanto, podían promocionar de curso.

El grupo con BR quedó formado por 115 estudiantes (63 chicos y 52 chicas) y el grupo con NR por 117 estudiantes (61 chicos y 56 chicas). No se encontraron diferencias significativas entre los grupos en función de la variable sexo $\chi 2(1, N=117)=.163 ; p=.686$.

\section{Instrumentos}

Para cada uno de los instrumentos utilizados, se realizó con la muestra de este estudio los análisis de fiabilidad y validez de constructo, los cuales se presentan en cada uno de ellos.

Para recoger la información sobre las metas académicas, se administró el Cuestionario de Metas Académicas (C.M.A.) de Núñez y González-Pienda (1994), el cual es una traducción al español del elaborado por Hayamizu y Weiner (1991). El cuestionario consta de 20 ítems, agrupados en tres categorías (metas de aprendizaje, metas de logro y metas de valoración social), con cinco alternativas de respuesta, siendo: 1 nunca, 2 raramente, 3 algunas veces, 4 frecuentemente y 5 siempre. La prueba $\mathrm{KMO}$ dio un valor de .826 y la prueba de esfericidad de Bartlett fue significativa ( $\mathrm{p}<.001)$. El Análisis de Componentes Principales arrojó tres factores que explicaban el $61.48 \%$ de la varianza total. La fiabilidad mediante $\alpha$ de Cronbach fue de .90 para el total de la escala, un $\alpha=.90$ para la subescala metas de aprendizaje, un $\alpha=.92$ para la subescala metas de logro y un $\alpha=.83$ para la subescala de valoración social.

Para medir las atribuciones causales se utilizó la subescala de Evaluación de Atribuciones Causales y Multidimensionales (EACM), la cual forma parte del Sistema Integrado de Evaluación de Atribuciones Causales y Procesos de Aprendizaje (SIACEPA) (Barca, 2000). La subescala EACM está formada por 24 ítems, agrupados en 7 factores, los cuales se responden mediante cinco alternativas: 1 totalmente en desacuerdo, 2 en desacuerdo, 3 más de acuerdo que en desacuerdo, 4 de acuerdo y 5 totalmente de acuerdo. La prueba KMO dio un valor de .802 y la prueba de esfericidad de Bartlett fue significativa $(\mathrm{p}<.001)$. El Análisis de Componentes Principales arrojó una estructura de siete factores que explicaban el $62.39 \%$ de la varianza total. La fiabilidad total, utilizando $\alpha$ de Cronbach, fue de .745. Para cada uno de los siete factores se obtuvieron los siguientes resultados respecto a su fiabilidad: atribución a la capacidad del alto rendimiento académico $(\alpha=.704)$, atribución a la suerte del rendimiento académico $(\alpha=.704)$, atribución al escaso esfuerzo del bajo rendimiento académico $(\alpha=.745)$, atribución al profesorado del bajo rendimiento académico $(\alpha=.699)$, atribución a la capacidad del bajo rendimiento académico $(\alpha=.712)$, atribución al esfuerzo del alto rendimiento académico $(\alpha=.687)$ y atribución a la facilidad de las materias del alto rendimiento académico $(\alpha=.701)$.

Para recoger información sobre las percepciones del apoyo familiar afectivo, se utilizó una adaptación de la escala de Figuera, Darias y Forner (2003). El instrumento original mide el apoyo familiar afectivo en la realización de estudios universitarios, pero Santana, Feliciano y Jiménez (2016) lo adaptaron para su uso en estudiantes de E.S.O. 
La escala contiene cuatro ítems con 4 alternativas de respuesta, siendo 1 totalmente en desacuerdo, 2 en desacuerdo, 3 de acuerdo y 4 totalmente de acuerdo. La prueba KMO dio un valor de .748 y la prueba de esfericidad de Bartlett fue significativa $(p<.001)$. El Análisis de Componentes Principales generó un solo factor que explicó el $68.32 \%$ de la varianza total. El $\alpha$ de Cronbach para esta muestra fue de .72.

Para analizar las variables relacionadas con el nivel socioeconómico, así como las expectativas familiares sobre el rendimiento de sus hijos, se diseñó un cuestionario que recogía información sobre variables sociodemográficas de identificación, nivel de formación del padre y madre, situación laboral del padre y la madre y dos preguntas sobre las expectativas del padre y de la madre respecto al nivel académico a alcanzar por el/la hijo/a.

\section{Procedimiento de recogida de información}

En primer lugar, se contactó con los Servicios Sociales de los distintos municipios de Tenerife para solicitar datos sobre los menores en situación de riesgo. Para garantizar algunos datos confidenciales de los menores y sus familias, no fue posible obtener las causas que motivaron la declaración de la situación de riesgo de los estudiantes de la muestra. Con los datos aportados se contactó con las familias de los menores a los cuales se les informó de la finalidad del estudio y se les solicitó su consentimiento para administrar los instrumentos a sus hijos. Los participantes fueron informados del propósito del trabajo y de preservar cualquier dato que pueda identificar su situación o la de sus familias. La aplicación de los instrumentos se llevó a cabo de manera individual, en dependencias sociales de los municipios, fuera del horario escolar, y siempre en presencia del padre o madre del menor. La recogida de información se realizó entre los meses de junio y septiembre de 2016.

\section{Procedimiento de análisis de datos}

Para las variables de naturaleza continua se llevaron a cabo, en primer lugar, análisis de correlaciones mediante el coeficiente de Pearson. Posteriormente, con el fin de establecer las diferencias entre los grupos en función de las variables utilizadas se llevó a cabo un MANOVA mediante modelo lineal general, utilizando como variable independiente intersujeto: rendimiento académico (bajo vs. normal/buen rendimiento) y como variables dependientes las metas académicas, atribuciones causales, y apoyo familiar afectivo. Como índice del tamaño del efecto usamos eta cuadrado parcial $\left(\eta^{2}\right)$.

Para las variables de naturaleza categórica se realizaron tablas de contingencias usando $\chi^{2}$ y los residuos tipificados corregidos, adoptando un intervalo de confianza de .95, por lo que valores superiores a 1.96 e inferiores a -1.96 se interpretaron como significativos. Los datos fueron analizados con el paquete estadístico SPSS (versión 21 para Windows).

\section{Resultados}

La Tabla 1 y la Tabla 2 recogen las correlaciones entre las puntuaciones obtenidas por el grupo de BR y NR respectivamente, en las variables: metas académicas (metas de aprendizaje, metas de logro, metas de valoración social), apoyo familiar afectivo y a las atribuciones causales del rendimiento académico. 
Tabla 1

Relación entre las variables medidas en el grupo de bajo rendimiento académico

\begin{tabular}{lccccccccccc}
\hline \multicolumn{10}{c}{ Variables } \\
\hline & MA & ML & MS & AF & AA & AS & AB & AP & AC & AE & AM \\
\hline MA & 1 & $.701^{* *}$ & $.506^{* *}$ & -.002 & $.202^{* *}$ & $-.259^{* *}$ & .09 & $-.356^{* *}$ & $-.245^{* *}$ & $.319^{* *}$ & .001 \\
ML & & 1 & $.506^{* *}$ & $.223^{*}$ & $.230^{*}$ & $-.346^{* *}$ & .14 & $-.271^{* *}$ & -.18 & $.464^{* *}$ & -.134 \\
MS & & & 1 & -.061 & -.053 & -.123 & $.301^{* *}$ & .135 & $.194^{*}$ & $.485^{* *}$ & -.055 \\
AF & & & & 1 & -.085 & $-.368^{* *}$ & $-.24^{* *}$ & -.177 & $-.219^{*}$ & -.05 & -.169 \\
AA & & & & & 1 & .045 & .136 & -.182 & $-.22^{*}$ & $.249^{* *}$ & $.289^{* *}$ \\
AS & & & & & 1 & .173 & $.429^{* *}$ & $.348^{* *}$ & -.188 & $.529^{* *}$ \\
AB & & & & & & 1 & .182 & $.283^{* *}$ & $.375^{* *}$ & .17 \\
AP & & & & & & & 1 & $.438^{* *}$ & .072 & $.404^{* *}$ \\
AC & & & & & & & & 1 & .06 & .055 \\
AE & & & & & & & & & 1 & -.005 \\
AM & & & & & & & & & & 1 \\
\hline
\end{tabular}

Nota: ${ }^{*} p<.05 ;{ }^{* *} p<.001$. MA: metas de aprendizaje; ML: metas de logro; MS: metas de valoración social; AF: apoyo familiar afectivo; AA: atribución a la capacidad del alto rendimiento; AS: atribución a la suerte del rendimiento; $\mathrm{AB}$ : atribución al escaso esfuerzo del bajo rendimiento; AP: atribución al profesorado del bajo rendimiento; AC: atribución a la capacidad del bajo rendimiento; AE: atribución al esfuerzo del alto rendimiento; AM: atribución a la facilidad de las materias del alto rendimiento.

Tabla 2

Correlaciones en las variables medidas en el grupo de alto rendimiento académico

\begin{tabular}{|c|c|c|c|c|c|c|c|c|c|c|c|}
\hline \multicolumn{12}{|c|}{ Variables } \\
\hline & MA & ML & MS & $\mathrm{AF}$ & $\mathrm{AA}$ & AS & $\mathrm{AB}$ & $\mathrm{AP}$ & $\mathrm{AC}$ & $\mathrm{AE}$ & $\mathrm{AM}$ \\
\hline MA & 1 & $.241^{* * *}$ & .144 & $.256^{* *}$ & -.11 & -.152 & $-.273^{* *}$ & -.163 & $-.394^{* * *}$ & $.219^{*}$ & -.024 \\
\hline ML & & 1 & -.067 & .078 & $.324^{* *}$ & $-.394^{* *}$ & $.392^{* *}$ & $-.283^{* *}$ & $-.226^{*}$ & $.299^{* * *}$ & -.075 \\
\hline MS & & & 1 & $.246^{* *}$ & .067 & $.306^{* *}$ & -.095 & $.209^{*}$ & $.228^{*}$ & .082 & $.209^{*}$ \\
\hline $\mathrm{AF}$ & & & & 1 & .089 & $.194^{*}$ & -.03 & .097 & -.055 & .096 & .07 \\
\hline AA & & & & & 1 & -.137 & $.408^{* *}$ & $-.192^{*}$ & $-.331^{* *}$ & $.465^{* *}$ & -.103 \\
\hline AS & & & & & & 1 & $-.367^{* *}$ & $.435^{* *}$ & $.371^{* *}$ & $-.314^{* *}$ & $.455^{* *}$ \\
\hline $\mathrm{AB}$ & & & & & & & 1 & $-.543^{* *}$ & .054 & $.259^{* *}$ & -.127 \\
\hline $\mathrm{AP}$ & & & & & & & & 1 & .108 & -.172 & $.419^{* *}$ \\
\hline $\mathrm{AC}$ & & & & & & & & & 1 & $-.368^{* *}$ & .179 \\
\hline $\mathrm{AE}$ & & & & & & & & & & 1 & .145 \\
\hline $\mathrm{AM}$ & & & & & & & & & & & 1 \\
\hline
\end{tabular}

Nota: ${ }^{*} p<.05 ;{ }^{* *} p<.001$. MA: metas de aprendizaje; ML: metas de logro; MS: metas de valoración social; AF: apoyo familiar afectivo; AA: atribución a la capacidad del alto rendimiento; AS: atribución a la suerte del rendimiento; AB: atribución al escaso esfuerzo del bajo rendimiento; AP: atribución al profesorado del bajo rendimiento; AC: atribución a la capacidad del bajo rendimiento; AE: atribución al esfuerzo del alto rendimiento; AM: atribución a la facilidad de las materias del alto rendimiento. 
Los resultados del MANOVA mostraron diferencias significativas entre los grupos F $(69,162)=38.5 p<.001, \eta^{2}=.943$. Para averiguar en qué variables se producían las diferencias, realizamos contrastes univariados (ANOVA) para cada una de las variables medidas. Los análisis arrojaron que existían diferencias significativas en todas las variables. Específicamente, como se puede observar en la Tabla 3, el grupo de estudiante en situación de riesgo con BR obtuvo puntuaciones más bajas en las metas académicas, tanto en las metas de aprendizaje, como en las metas de logro y en las de valoración social, así como una menor puntuación en apoyo familiar afectivo.

Respecto a las atribuciones causales del rendimiento académico el grupo de BR se caracterizó por tener puntuaciones más bajas en atribución a la capacidad del alto rendimiento, atribución al escaso esfuerzo del bajo rendimiento, atribución al profesorado del bajo rendimiento y atribución al esfuerzo del alto rendimiento. Por el contrario, obtuvo puntuaciones más altas en atribución a la suerte del rendimiento, atribución a la capacidad del bajo rendimiento y atribución a la facilidad de las materias del alto rendimiento académico.

Tabla 3

Medias, desviaciones típicas y significación de las variables personales en función de los grupos

\begin{tabular}{|c|c|c|c|c|c|c|c|}
\hline \multirow{3}{*}{ VARIABLES } & \multicolumn{4}{|c|}{$\begin{array}{c}\text { Grupos } \\
\text { (situación de riesgo) }\end{array}$} & \multirow{3}{*}{$\begin{array}{c}F \\
(8,223)\end{array}$} & \multirow{3}{*}{$\mathrm{p}$} & \multirow{3}{*}{$\eta^{2}$} \\
\hline & \multicolumn{2}{|c|}{ NR } & \multicolumn{2}{|c|}{ BR } & & & \\
\hline & $\mathrm{M}$ & DT & $\mathrm{M}$ & DT & & & \\
\hline \multicolumn{8}{|l|}{ METAS ACADÉMICAS } \\
\hline De aprendizaje & 3.25 & 0.96 & 2.65 & 0.95 & 10.65 & $<.001$ & .089 \\
\hline De logro & 4.55 & 0.57 & 3.7 & 1.18 & 7.47 & $<.001$ & .17 \\
\hline De valoración social & 2.59 & 1.09 & 2.57 & 0.94 & 5.25 & $<.001$ & .000 \\
\hline APOYO FAMILIAR AFECTIVO & 3.55 & 0.57 & 3.1 & 0.85 & 7.61 & $<.001$ & .086 \\
\hline \multicolumn{8}{|l|}{ ATRIBUCIONES CAUSALES } \\
\hline A la capacidad (alto rendimiento) & 3.83 & 0.94 & 3.57 & 1.28 & 3.8 & $<.001$ & .13 \\
\hline A la capacidad (bajo rendimiento) & 2.88 & 1.03 & 2.97 & 0.94 & 1.92 & $<.05$ & .000 \\
\hline A la suerte & 2.19 & 0.71 & 2.51 & 0.7 & 4.74 & $<.001$ & .051 \\
\hline Al escaso esfuerzo (bajo rendimiento) & 3.97 & 0.68 & 3.78 & 0.81 & 2.91 & $<.001$ & .016 \\
\hline Al esfuerzo (alto rendimiento) & 4.02 & 0.63 & 3.55 & 0.71 & 5.96 & $<.001$ & .112 \\
\hline Al profesorado (bajo rendimiento) & 2.96 & 0.71 & 2.85 & 0.79 & 4.66 & $<.001$ & .000 \\
\hline A la facilidad de las materias (alto rendimiento) & 2.98 & 0.96 & 3.04 & 1.02 & 3.87 & $<.001$ & .000 \\
\hline
\end{tabular}

Respecto a las variables categóricas, para el nivel de estudio de los progenitores, en la Tabla 4 se exponen los resultados en función del grupo. Se obtuvieron diferencias significativas para el nivel educativo del padre, pero no para el de la madre. Los residuos tipificados corregidos mostraron que el porcentaje de padres con titulación universitaria fue significativamente mayor en el grupo del alumnado con NR. 
Tabla 4

Nivel educativo del padre y de la madre en función de los grupos

\begin{tabular}{llcc}
\hline \multirow{2}{*}{ Nivel educativo } & \multicolumn{2}{c}{$\begin{array}{c}\text { Grupos } \\
\text { (situación de riesgo) }\end{array}$} \\
\cline { 3 - 4 } & & NR (\%) & BR (\%) \\
\cline { 2 - 3 } Padre estudios & 12.8 & 17.4 \\
& Graduado escolar & 26.5 & 28.7 \\
& FP & 13.7 & 17.4 \\
& Bachillerato & 16.2 & 18.3 \\
& COU/PAU & 12.8 & 13.9 \\
& Titulación universitaria & 17.9 & 4.3 \\
\cline { 2 - 4 } Madre & Sin estudios & 12.8 & 11.3 \\
& Graduado escolar & 28.2 & 37.4 \\
& FP & 8.5 & 17.4 \\
& Bachillerato & 19.7 & 12.2 \\
& COU/PAU & 10.3 & 5.2 \\
& Titulación universitaria & 20.5 & 16.5 \\
\hline
\end{tabular}

Respecto a la situación laboral del padre y de la madre, se obtuvieron diferencias significativas entre los grupos en ambos progenitores. En el caso de la situación laboral del padre, los residuos tipificados corregidos dieron dos diferencias. El grupo con NR tenía un porcentaje mayor de padres funcionarios, mientras que en el grupo de BR la mayoría de los padres trabajaban por cuenta propia. Respecto a la situación de las madres, los residuos tipificados corregidos fueron coincidentes con los anteriores datos. Fue el grupo con NR las que tenían un mayor porcentaje de madres funcionarias, mientras que en el grupo con BR el porcentaje de madres en situación de desempleo era mayor. (Ver Tabla 5).

Tabla 5

Situación laboral del padre y de la madre en cada grupo

\begin{tabular}{|c|c|c|c|}
\hline \multicolumn{2}{|l|}{ Situación laboral } & \multicolumn{2}{|c|}{$\begin{array}{c}\text { Grupos } \\
\text { (situación de riesgo) }\end{array}$} \\
\hline \multirow{7}{*}{ Padre } & & NR (\%) & BR (\%) \\
\hline & Ayuda básica & 12 & 10.4 \\
\hline & Desempleado & 24.8 & 17.4 \\
\hline & Pensionista & 1.7 & 1.7 \\
\hline & Funcionario & 6 & -- \\
\hline & Trabaja por cuenta ajena & 43.6 & 41.7 \\
\hline & Trabaja por cuenta propia & 12 & 28.7 \\
\hline
\end{tabular}




\begin{tabular}{llll}
\cline { 2 - 3 } Myuda básica & 13.7 & 10.4 \\
Desempleada & 27.4 & 39.1 \\
Pensionista & -- & 1.7 \\
& Funcionaria & 8.5 & -- \\
Trabaja por cuenta ajena & 41 & 39.1 \\
& Trabaja por cuenta propia & 9.4 & 9.6 \\
\hline
\end{tabular}

Por último, en la Tabla 6 se muestran los resultados obtenidos en la expectativa de logro que tenían los padres y madres sobre el futuro académico de su hijo/a. En el caso de las expectativas del padre, el análisis estadístico $\chi^{2}$ no mostró diferencias significativas entre los grupos. Respecto a las expectativas de la madre, sí que encontraron diferencias estadísticas significativas. Según los residuos tipificados corregidos, el grupo de alumnos con NR se caracterizó por tener un porcentaje mayor de madres que tenían expectativas académicas universitarias para sus hijos/as. Mientras que en el grupo de alumnos con BR se observó una predominancia de madres que esperaban que como máximo sus hijos finalicen la E.S.O.

Tabla 6

Expectativas de padres y madres sobre el futuro académico de su hijo o hija

\begin{tabular}{clcc}
\hline \multirow{2}{*}{ Nivel expectativas } & \multicolumn{2}{c}{$\begin{array}{c}\text { Grupos } \\
\text { (situación de riesgo) }\end{array}$} \\
\cline { 3 - 3 } & & NR (\%) & BR (\%) \\
\cline { 2 - 3 } Padre & E.S.O. & 4.3 & 7 \\
& FP & 1.7 & 7.8 \\
& Bachillerato & 2.6 & 6.1 \\
& Titulación universitaria & 71.8 & 58.3 \\
& No sabe & 19.7 & 20.9 \\
& & 2.6 & 13.9 \\
Madre & & 4.3 & 0.9 \\
& E.S.O. & 0.9 & 7 \\
& FP & 76.1 & 54.8 \\
& Bachillerato & 16.2 & 23.5 \\
\hline
\end{tabular}

\section{Discusión y conclusiones}

El objetivo principal de este estudio fue comprobar las diferencias existentes entre el alumnado de E.S.O., declarado en situación de riesgo, con alto y bajo rendimiento académico según sus variables personales y sociofamiliares. A nivel global, los resultados obtenidos permiten afirmar que existían diferencias entre los estudiantes con NR y BR tanto en las variables personales (metas académicas, atribuciones causales del rendimiento académico y apoyo familiar afectivo) como en las sociofamiliares consideradas 
en este trabajo. En contraste con el grupo de estudiantes en riesgo con BR, fueron los estudiantes en riesgo con NR los que presentaron valores más adaptativos para su rendimiento académico en los factores analizados, los cuales pueden estar actuando como factores protectores de su escolarización (Rogero, 2014).

De especial relevancia resultaron las relaciones encontradas entre las diferentes variables analizadas en función del rendimiento de estos estudiantes. Las relaciones halladas permiten concluir que en las metas académicas ambos grupos establecieron relaciones parecidas. Por lo que no podemos afirmar que haya un patrón de correlaciones más favorecedor del rendimiento académico. Sin embargo, las relaciones obtenidas en las atribuciones causales permiten afirmar que en el grupo con alto rendimiento se estableció un patrón de relaciones que benefician el aprendizaje y rendimiento académico. El patrón de asociaciones de atribuciones causales con predominio de locus de control interno, encontrado en el alumnado con BR, es muy importante que se fomente también en los alumnos con bajo rendimiento, ya que investigaciones previas realizadas con poblaciones similares a la de este estudio, han mostrado que las personas con un locus de control interno están más capacitadas y más dispuestas a poner en marcha los cambios necesarios para abandonar su situación de riesgo (Juby \& Rycraft, 2004). Según estos resultados las atribuciones causales pueden actuar como factor protector de estos estudiantes.

Cuando se analizaron las diferencias entre los estudiantes declarados en riesgo en función de su rendimiento académico en las metas académicas, atribuciones causales, apoyo familiar afectivo y factores sociofamiliares, se encontró que existía un efecto significativo de la condición del grupo, y con un tamaño del efecto altamente significativo (94.3\% de la varianza explicada). Los análisis también mostraron que en todas las variables continuas (metas académicas, apoyo familiar afectivo y atribuciones causales) se daban diferencias significativas entre ambos grupos. En función de ello, y descartando aquellas variables que mostraron un tamaño del efecto insignificante, resulta muy importante resaltar que se dieron diferencias con un tamaño del efecto moderado para metas de aprendizaje, apoyo familiar afectivo y atribución al esfuerzo del alto rendimiento académico, y diferencias con un tamaño del efecto grande para metas de logro y atribución a la capacidad del alto rendimiento académico. Dándose además todas las diferencias en estas variables personales con valores superiores para el grupo de alto rendimiento académico, que se caracterizó, por tanto, por tener mayores metas de aprendizaje y de logro, una mayor percepción de apoyo familiar afectivo y atribución del buen rendimiento académico al esfuerzo y a su propia capacidad. Los resultados encontrados apoyan los hallazgos de otros estudios en los que se ha indagado la relación conjunta entre metas académicas y atribuciones causales como estrategias de mejora del aprendizaje y predictores del rendimiento (Shell \& Husman, 2008; Wolters, Fan \& Daugherty, 2013).

Respecto a la percepción que tenían estos estudiantes sobre el apoyo que reciben de sus familias, se comprobó que el alumnado con NR se sentía significativamente más apoyado que el grupo con BR. Estos resultados van en la línea de lo encontrado en estudios previos que señalan la importancia del apoyo familiar percibido para el buen rendimiento académico (Córdoba-Caro et al, 2011; Yamamoto \& Holloway, 2010). En consecuencia, los resultados de este estudio permiten concluir que, aunque el menor 
viva en un contexto familiar y social de riesgo, el que se sienta apoyado por su familia, contribuye a que a nivel académico tenga mayores probabilidades de éxito, tal y como se ha encontrado en otros estudios (Orthner, Jones-Sanpei \& Williamson, 2004).

Asimismo, las diferencias encontradas entre los grupos referidas a las variables sociofamiliares reflejaron que el grupo con NR se caracterizó por tener un mayor porcentaje de padres con estudios universitarios, así como padres con una mayor estabilidad laboral (funcionarios) y con madres con expectativas académicas más altas para sus hijos. En cambio, en el grupo con BR se observó que tenían un mayor porcentaje de padres que trabajan por cuenta propia, madres en desempleo y con expectativas académicas más a bajas. Sin duda, estos hallazgos evidencian que las situaciones laborales de los padres asociadas a una mayor inestabilidad o precariedad laboral, como el desempleo o ser trabajador autónomo (Laparra, 2006), convierten a estos estudiantes en más vulnerables a la hora de tener éxito académico y no abandonar prematuramente la educación obligatoria (Spera, Wentzel \& Matto, 2009). Además apoyan las conclusiones señaladas por otros autores realizadas con estudiantes de la E.S.O, aunque no declarados en situación de riesgo (Córdoba-Caro et al., 2011). En el caso del alumnado declarado en riesgo, reflejan la importancia de su inclusión, ya que pueden servir como base para el desarrollo de acciones o programas con las familias de estos estudiantes. No obstante, es importante señalar que para poder determinar con mayor precisión la influencia de los factores sociales en muestras similares a la de este estudio, futuros trabajos deberían tener en cuenta no solo la situación laboral, sino los ingresos económicos de la unidad familiar como otro factor del nivel social de los padres con incidencia en la situación académica de los estudiantes (Fitzpatrick, McKinnon, Blair \& Willoughby, 2014).

Respecto a las expectativas parentales sobre el estudio de sus hijos, aunque solo encontramos diferencias significativas entre los grupos en las expectativas de las madres, los resultados son consistentes y van en la dirección de los obtenidos en otras investigaciones en los que se ha mostrado una contrastada asociación entre las expectativas de los padres y el rendimiento académico (Wood, Kaplan \& McLoyd, 2007) ya que la gran mayoría de padres y madres de este estudio esperaban que sus hijos/as alcanzaran el nivel académico más alto.

Tomados en su conjunto estos resultados nos ofrecen indicios de la importancia que recobra en estudiantes declarados en situación de riesgo, identificar los factores personales y sociofamiliares que pueden estar incidiendo en su rendimiento académico. Aunque estos hallazgos no pueden generalizarse a todos los estudiantes declarados en esta situación, y algunas de las limitaciones de este estudio deben ser consideradas en futuros trabajos, dentro de las cuales resaltamos disponer de muestras más amplias, pertenecientes a otros contextos geográficos, abren futuras líneas de investigación e intervenciones educativas en una de las poblaciones escolares que puede presentar mayores indicadores de riesgo de bajo rendimiento y abandono prematuro de los estudios (Berger, 2004).

El hecho de que estos estudiantes finalicen la E.S.O., puede ser en sí mismo un factor protector que les ayude a salir de la situación de riesgo personal y social en la que viven (Rogero, 2014). No siempre la escuela cuenta con los medios y recursos para influir o modificar las variables sociofamiliares de estos estudiantes, pero promover en 
ellos unas altas metas académicas y unas atribuciones causales del rendimiento asociadas a su propio esfuerzo y capacidad, contribuiría a mitigar la influencia de muchas de las desventajas, asociadas al contexto social o familiar, con las que conviven. Por ello, futuras investigaciones deberían seguir indagando sobre otros posibles factores que permitan determinar y discriminar las diferencias de rendimiento de estos estudiantes. Además, si queremos garantizar una integración social y laboral efectiva en la edad adulta de los menores que se encuentran actualmente en situación de riesgo, hay que abogar por investigaciones que deriven en intervenciones interdisciplinares, con implicación de los servicios sociales, jurídicos y, principalmente educativos, que posibiliten actuaciones específicas en unos estudiantes que por su situación de doble vulnerabilidad (situación de riesgo y bajo rendimiento escolar) se encuentran más desfavorecidos en los centros.

\section{Referencias}

Alderman, M. K. (2008). Motivation for achievement: Possibilities for teaching and learning. Nueva York: Taylor \& Francis.

Ames, C. \& Archer, J. (1987). Mother's beliefs about the role of ability and effort in school learning. Journal of Educational Psychology, 18, 409-414.

Barca, A. (2000). Escala SIACEPA: Sistema Integrado de Evaluación de Atribuciones Causales y Enfoques de Aprendizaje para el Alumnado de Educación Secundaria. Técnicas de Intervención Psicoeducativa. A Coruña: Monografías y Publicaciones de la Revista GalegoPortuguesa de Psicoloxía e Educación, Universidade da Coruña/Universidade do Minho/Consellería de Educación e Ordenación Universitaria. Xunta de Galicia.

Benner, A., \& Mistry, R. (2007). Congruence of mother and teacher educational expectations and low-income youth's academic competence. Journal of Educational Psychology, 99, 140-153. doi: 10.1037/0022-0663.99.1.140

Benzies, K., \& Mychasiuk, R. (2009). Fostering family resilience: A review of the key protective factors. Child and Family Social Work, 14(1), 103-114. doi: 10.1111/j.13652206.2008.00586.x

Berger, K.S. (2004). Psicología del Desarrollo: Infancia y Adolescencia. (6 $6^{\mathbf{a}}$ Ed.). Madrid: Editorial Médica Panamericana.

Brown, L., \& Iyengar, S. (2008). Parenting styles: The impact on student achievement. Marriage and Family Review, 43, 14-38. doi: 10.1080/01494920802010140

Buehler, C., \& Gerard, J. M. (2013). Cumulative family risk predicts increases in adjustment difficulties across early adolescence. Journal of Youth and Adolescence, 42(6), 905-920. doi: 10.1007/s10964-012-9806-3

Cabanach, R., Valle, A., Herpe, M., Rodriguez, S., Pinero, I., \& Rosario, P. (2009). Diseño y validación de un Cuestionario de Gestión Motivacional. Revista de Psicodidáctica, 14(1), 29-47. Recuperado de http://www.ehu.eus/ojs/index.php/psicodidactica/article/ view/249

Chen, W., \& Gregory, A. (2009). Parental involvement as a protective factor during the transition to high school. Journal of Educational Research, 103, 53-62. doi: $10.1080 / 00220670903231250$ 
Closas, A., Sanz, M., \& Ugarte, M. (2011). An explanatory model of the relations between cognitive and motivational variables and academic goals. Revista de Psicodidáctica, 16(1), 19-38. Recuperado de http://www.ehu.eus/ojs/index.php/psicodidactica/article/ view/1142/782

Córdoba-Caro, L., Preciado, V., Pérez, L., Carrizosa, M., \& Molina, S. (2011). Determinantes socioculturales: su relación con el rendimiento académico en alumnos de Enseñanza Secundaria Obligatoria. Revista de Investigación Educativa, 29(1), 83-96. Recuperado de http://revistas.um.es/rie/article/view/110361/126942

Davis-Kean, P. (2005). The influence of parent education and family income on child achievement: The indirect role of parental expectations and the home environment. Journal of Family Psychology, 19(2), 294-304. doi: 10.1037/0893-3200.19.2.294

Deci, E. \& Ryan, R. (1985). Intrinsic motivation and self-determination in human behavior. Nueva York: Plenum.

De la Orden, D. \& González, C. (2005). Variables que discriminan entre alumnos de bajo y medio-alto rendimiento académico. Revista de Investigación Educativa, 23 (2), 573-599. Recuperado de http://revistas.um.es/rie/article/view/98261

Dweck, C., \& Elliot, D. (1983). Achievement motivation. En P.H. Mussen \& E. M. Hetherington (Eds.), Handbook of child psychology (vol. IV, pp. 643-691) Nueva York: Wiley.

Entwistle, N. \& Kozéki, B. (1985). Relationships between school motivation, approaches to studying and attainment among british and hungarian adolescents. British Journal of Educational Psychology, 55, 124-137.

Figuera, P., Darias, I. \& Forner, A. (2003). Las competencias académicas previas y el apoyo familiar en la transición a la Universidad. Revista de Investigación Educativa, 21(2), 349-369. Recuperado de http://revistas.um.es/rie/article/view/99251

Fitzpatrick, C., McKinnon, R. D., Blair, C. B., \& Willoughby, M. T. (2014). Do preschool executive function skills explain the school readiness gap between advantaged and disadvantaged children? Learning and Instruction, 30, 25-21.

doi: 10.1016/j.learninstruc.2013.11.003

Gordon, M. S., \& Cui, M. (2012). The Effect of School-Specific Parenting Processes on Academic Achievement in Adolescence and Young Adulthood. Family Relations, 61, 728-741. doi: 10.1111/j.1741-3729.2012.00733.x

Hayamizu, T., \& Weiner, B. (1991). A test Dweck' model of achievement goals as related to perceptions of ability. Journal of Experimental Education, 59, 226-234. doi https:// doi.org/10.1080/00220973.1991.10806562

Jackson, S., Ajayi, S., \& Quigley, M. (2003). By Degrees: The first year. Londres: National Children's Bureau and the Frank Buttle Trust.

Juby, C., \& Rycraft, J. R. (2004). Family preservation strategies for families in poverty. Families in Society, 85(4), 581-587. doi: http://dx.doi.org/10.1606/1044-3894.1847

Ley Orgánica 8/2013, de 9 de diciembre, para la mejora de la calidad educativa. Boletín Oficial del Estado, de 10 de diciembre de 2013, 295

Ley Orgánica 8/2015 de 22 de julio, de modificación del sistema de protección a la infancia y a la adolescencia. Boletín Oficial del Estado de 23 de julio de 2015, 175.

Ley 26/2015, de 28 de julio, de modificación del sistema de protección a la infancia y a la adolescencia. Boletín Oficial del Estado de 29 de julio de 2015, 180. 
Laparra, M. (2006). La construcción del empleo precario: dimensiones, causas y tendencias de la precariedad laboral. Madrid: Caritas Española y Fundación Foessa.

Martínez, R. A., \& Álvarez, L. (2005). Fracaso y abandono escolar en educación secundaria obligatoria: implicación de las familias y de los centros escolares. Aula Abierta, 85, 127-146. Recuperado de https://www.unioviedo.es/reunido/index.php/AA/issue/ viewFile/1033/131

McKenzie, K., Gow, K., \& Schweitzer, R. (2004). Exploring first-year academic achievement through structural equation modelling. Higher Education Research \& Development, 23(1), 95-112. doi: https://doi.org/10.1080/0729436032000168513

McLoyd, V.C., Kaplan, R., Purtell, K., Bagley, E., Hardaway, C., \& Smalls, C. (2009). Poverty and socioeconomic disadvantage in adolescence. En R. M. Lerner \& L. Steinberg (Eds.), Handbook of adolescent psychology (3 ${ }^{\mathrm{a}}$ ed., vol. 2, pp. 444-491). Hoboken, NJ: John Wiley \& Sons. doi: 10.1002/9780470479193.adlpsy002014

Meece, J. L., Anderman, E.L., \& Anderman, L.H. (2006). Classroom goal structure, student motivation, and academic achievement. American Review of Psychology, 57, 487-503. doi: 10.1146/annurev.psych.56.091103.070258

Mistry, R., White, E., Benner, A., \& Huynh, V. (2009). A longitudinal study of the simultaneous influence of mothers' and teachers' educational expectations on lowincome youth's academic achievement. Journal of Youth and Adolescence, 38, 826-838. doi: 10.1007/s10964-008-9300-0

Naranjo, M. L. (2009). Motivación: Perspectivas teóricas y algunas consideraciones de su importancia en el ámbito educativo. Revista Educación, 33(2), 153-170. doi: 10.15517/revedu.v33i2.510

Núñez, J. C., \& González-Pienda, J. A. (1994). Determinantes del rendimiento escolar. Oviedo: Universidad de Oviedo.

Oliva, A., Jiménez, J. M., Parra, A., \& Sánchez-Queija, I. (2008). Acontecimientos vitales estresantes, resiliencia y ajuste adolescente. Revista de Psicopatología y Psicología Clínica, 13(1), 53-62. doi: 10.5944/rppc.vol13.num.1.2008.4050

Orthner, D. K., Jones-Sanpei, H., \& Williamson, S. (2004). The resilience and strengths of low-income families. Family Relations, 53(2), 159-167. doi: 10.1111/j.00222445.2004.00006.x

Pintrich, P. \& De Groot, E. (1990). Motivational and self-regulated learning components of classroom performance. Journal of Educational Psychology, 82(1), 33-40. Recuperado de http://rhartshorne.com/fall-2012/eme6507-rh/cdisturco/eme6507-eportfolio/ documents/pintrich\%20and\%20degroodt\%201990.pdf

Rao N., Moely B., \& Sachs J. (2000). Motivational Beliefs, Study Strategies, and Mathematics Attainment in High- and Low-Achieving Chinese Secondary School Students. Contemporary Educational Psychology, 25(3), 287-316. doi: https://doi.org/10.1006/ ceps.1999.1003

Rogero, J. (2012). El fracaso escolar, causas y alternativas. En la calle: revista sobre situaciones de riesgo social, 21, 9-13. Recuperado de https://dialnet.unirioja.es/servlet/ articulo? codigo $=3832080$

Rogero, J. (2014). Éxito escolar - Éxito social y los excluidos del éxito. Revista nacional e internacional de educación inclusiva, 7(2), 36-52. Recuperado de http://www.revistaeducacioninclusiva.es/index.php/REI/article/view/144 
Ross, C. E., \& Mirowsky, J. (2013). The sense of personal control: Social structural causes and emotional consequences. En C. S. Anshensel, J. C. Phelan \& A. Bierman (Eds.), Handbook of the sociology of mental health (pp. 379-402). Países Bajos: Springer.

Roy, A.L., \& Raver, C.C. (2014). Are all risks equal? Early experiences of poverty-related risk and children's functioning. Journal of Family Psychology, 28(3), 391-400. doi: $10.1037 / \mathrm{a} 0036683$

Santana, L., Feliciano, L., \& Jiménez, A. (2016). Apoyo familiar percibido y proyecto de vida del alumnado inmigrante de Educación Secundaria. Revista de Educación, 372, 35-62. doi: 10.4438/1988-592X-RE-2015-372-314

Schoon, I., Bynner, J., Joshi, H-. Parson, S., Wiggins, R., \& Sacker, A. (2002). The influence of context, timing, and duration of risk experiences for the passage from childhood to midadulthood. Child Development, 73(5), 1486-1504. doi: https://doi. org/10.1111/1467-8624.00485

Serna, C., Yubero, S., \& Larrañaga, E. (2008). Exclusión educativa y social: el contexto social como escenario del fracaso escolar. Bits: Boletín informativo trabajo social, 13. Recuperado de https://dialnet.unirioja.es/ejemplar/219419

Shell, D. F., \& Husman, J. (2008). Control, Motivation, Affect, and Strategic Self-Regulation in the College Classroom: A Multidimensional Phenomenom. A Journal of Educational Psychology, 100(2), 443-459. doi: 10.1037/0022-0663.100.2.443

Spera, C., Wentzel, K., \& Matto, H. (2009). Parental aspirations for their children's educational attainment: relations to ethnicity, parental education, children's academic performance, and parental perceptions of school climate. Journal of Youth and Adolescence, 38, 1140-1152. doi: 10.1007/s10964-008-9314-7

Stein, M., \& Munro, E. R. (2008). Comparative exploration of care leavers transitions to adulthood: An introduction. En M. Stein \& E.R. Munro (Eds.), Young people's transitions from care to adulthood International research and practice (pp.11-21). Londres: Jessica Kingsley

Whipple, S.S., Evans, G.W., Barry, R.L., \& Maxwell, L.E. (2010). An ecological perspective on cumulative school and neighborhood risk factors related to achievement. Journal of Applied Developmental Psychology, 31(6), 422-427. doi: 10.1016/j.appdev.2010.07.002

Wolters, C. A., Fan, W., \& Daugherty, S. G. (2013). Examining Achievement Goals and Causal Attributions Together as Predictors of Academic Functioning. The Journal of Experimental Education, 81(3), 295-321. doi: 10.1080/00220973.2012.700498

Wood, D., Kaplan, R., \& McLoyd, V.C. (2007). Gender Differences in the Educational Expectations of Urban, Low-Income African American Youth: The Role of Parents and the School. Journal of Youth and Adolescence, 36(4), 417-427. doi: 10.1007/s10964-007-9186-2.

Yamamoto, Y., \& Holloway, S. (2010). Parental expectations and children's academic performance in sociocultural context. Educational Psychology Review, 22, 189-214. doi: 10.1007/s10648-010-9121-z.

Fecha de recepción: 1 de septiembre de 2017.

Fecha de revisión: 18 de septiembre de 2017.

Fecha de aceptación: 15 de mayo de 2018. 